\title{
Importancia del diseño tecnopedagógico basado en el enfoque de la acción, para reforzar el dominio del idioma inglés como segunda lengua
}

\section{Importance of the technological design based on the Action approach to reinforce the fluency in English as second language}

\author{
Olga Andrea Pedroza Rojas ${ }^{1}$, Miguel Francisco Crespo Alvarado 2 iD \\ ${ }^{1}$ Maestría en e-Learning, Universidad Autónoma de Bucaramanga, Bucaramanga, Colombia \\ ${ }^{2}$ Grupo Interdisciplinario de Investigaciones Sistémico Interpretativas S.C., Torreón, México \\ opedroza@unab.edu.co, $\underline{\text { migcrespo@ @hotmail.com }}$
}

(Recibido: 5 Junio 2017; aceptado: 27 Septiembre 2017)

\begin{abstract}
Resumen. Este artículo presenta la importancia del diseño de aplicaciones tecnológicas que refuercen el aprendizaje del inglés como segunda lengua, con tal eficiencia que el usuario pueda aprobar un examen internacional de suficiencia. Se abordan, para ello, los siguientes temas: influencia del diseño tecnopedagógico en los procesos de refuerzo del inglés como lengua extranjera; importancia del enfoque de la acción para la creación de material educativo, basado en los parámetros de diseño propuestos por Tomlinson para perfeccionar el aprendizaje del inglés como lengua extranjera y en los lineamientos de diseño instruccional ADDIE para la creación de aplicaciones móviles. En la revisión bibliográfica se seleccionaron los documentos pertinentes para la temática, que indican que las Tecnologías de Información y Comunicación (TIC), especialmente el diseño tecnopedagógico, permiten mejorar el proceso enseñanza - aprendizaje de inglés como lengua extranjera, al ajustar el instrumento elaborado a las necesidades de los estudiantes y aplicarlo en las prácticas de docentes virtuales.
\end{abstract}

Palabras clave: materiales educativos, diseño tecnopedagógico, inglés como segunda lengua, tecnologías de información y comunicación, enfoque de la acción.

\begin{abstract}
This article shows the importance of technological apps design to reinforce the English as second language, so as to allow the pass of an international English exam. This article contains: the influence of the techno pedagogical design in the process of English as second language to pass an international exam, the importance of the Oriented Action Approach to create interactive material in the English as second language, the patterns of foreign language educative material design by Tomlinson. Previously, there was a bibliographical revision to verify the existence of limited investigations about this topic. This was linked to an intensive search to select very carefully the key documents, which gave evidence that a good use of the techno pedagogical design allows people to improve the teaching and learning English process fitted to the students' needs and interests in order to put it into practice in a virtual class.
\end{abstract}

Keywords: Material design, Technopedagogical design, Teaching, Learning and enhancing English language process, ICT, Oriented action approach. 


\section{Introducción}

La sociedad moderna se caracteriza por su creciente dependencia del desarrollo tecnológico, cuyo auge ha dinamizado las comunicaciones e impuesto la virtualidad como una herramienta casi de forzosa aplicación en la vida cotidiana. La relación entre enseñanza y aprendizaje se ha adaptado de manera gradual al uso de las tecnologías de información y comunicación, a la masificación de la educación y al cambio de medios y paradigmas educativos. Gracias a esta dinámica formativa impuesta por el apogeo tecnológico y comunicativo, los procesos formativos llegan hoy a los usuarios en sus propias casas o sitios de trabajo, sin necesidad de congregarse en un aula de clase y sin supeditarse a parceladas franjas de tiempo, de estricta rigidez. Esta dinámica ha creado la necesidad de idear, diseñar e implementar nuevas formas de aprendizaje y de desarrollar metodologías que potencien la capacidad autodidacta.

Adicionalmente, esta nueva realidad de un mundo tecnológicamente dependiente obliga a superar la barrera de los idiomas; el dominio de un segundo idioma se convierte en un asunto decisivo. En la actualidad, se considera que las TIC posibilitan no solo el refuerzo de los conocimientos adquiridos de una lengua extranjera, sino también el desarrollo de estrategias cognitivas y metacognitivas para la vida diaria. Con lo anterior, se puede comprender la creciente tendencia a la implementación estratégica de las TIC como un factor de especial relevancia en la creación de materiales ajustados a las necesidades e intereses de los estudiantes. Entre estas estrategias, especialmente el e-learning ${ }^{1}$ ha aportado una nueva perspectiva de educación para las sociedades contemporáneas: en el caso de la adquisición de una lengua extranjera, ha favorecido la creación de un ambiente virtual para que el aprendiz y el docente dispongan, analicen y evalúen el medio de enseñanza y los materiales desde un ángulo singular y logren un aprendizaje significativo. El presente artículo muestra las bondades del diseño de aplicaciones móviles, propias de la educación virtual, en los procesos de enseñanza y aprendizaje del inglés como lengua extranjera, enfocado a la presentación de un examen internacional de suficiencia. Se resalta, para ello, cómo el diseño tecnopedagógico y el enfoque de la acción pueden influir en los procesos de refuerzo del inglés como segunda lengua. Según señala Agudelo:

\section{El diseño instruccional nos lleva a reconocer en sus modelos una oportunidad para hacer efectivo el proceso de aprendizaje, ya que orientan la realización de una planificación adecuada que ayude a crear ambientes virtuales de aprendizaje, orientados al cumplimiento de objetivos de aprendizaje concretos [1].}

A partir de esta afirmación, se inicia la búsqueda de incorporación de las Apps y del Mobile Learning ${ }^{2}$ al refuerzo del inglés como segunda lengua, de modo que el nivel de dominio adquirido posibilite la aprobación de un examen internacional. Cerro [2] hizo énfasis en los beneficios del uso de las aplicaciones móviles educativas en el presente, especialmente de aquellas creadas para un aprendizaje personalizado. Sus reflexiones se orientaron a las ventajas que supone la flexibilidad de tales aplicaciones y al aporte en cada estilo de aprendizaje por parte de los estudiantes. El aporte de la aplicación de herramientas tecnológicas en los procesos virtuales de enseñanza y aprendizaje del inglés como lengua extranjera radica en ofrecer una técnica de aprendizaje confiable basada en estrategias metacognitivas. Este propósito hace que la labor del docente virtual sea la de un mediador, guía y facilitador que ofrece a los alumnos las orientaciones, instrumentos y mecanismos apropiados, para que ellos mismos creen el ambiente formativo y se sumerjan en él como centro de sus respectivos procesos de aprendizaje. Se hace énfasis en la necesidad de utilizar aplicaciones móviles, consideradas como objetos virtuales de aprendizaje, centradas en el estudiante y ricas en ejercicios, recomendaciones y retroalimentaciones inmediatas, para reforzar las habilidades lingüísticas que los alumnos requieran. Con certeza, el desarrollo de nuevas investigaciones sobre los resultados (eficiencia y eficacia) del método aquí expuesto, contrastado con otras técnicas de aprendizaje, permitirá su perfeccionamiento.

1 Término utilizado para referirse a la educación dada en un entorno digital, empleando la internet como medio para el proceso de enseñanza y aprendizaje.

2 Apps y Mobile Learning son una de las tendencias del uso de las TIC que permiten a los usuarios acceder a los contenidos y actividades por medio de recursos tecnológicos. "La metodología de enseñanza y aprendizaje es por medio de dispositivos portátiles que son conectados inalámbricamente para acceder a contenido que se encuentra accesible a través de Internet" [2]. 
El propósito de este artículo es, como se ha dicho, evidenciar la importancia de diseñar aplicaciones tecnológicas destinadas a reforzar el aprendizaje del inglés como segunda lengua, a niveles de suficiencia tales que permitan la aprobación de un examen internacional. Para lograr este propósito, se presenta la siguiente estructura procedimental: primero, se muestra el papel que cumplen las tecnologías de información y comunicación en el aprendizaje de una segunda lengua. Después, se aborda la influencia que ha tenido el diseño tecnopedagógico en los procesos de refuerzo del inglés como lengua extranjera. Luego, se discute sobre la importancia del enfoque de la acción para la creación de material interactivo en la búsqueda por desarrollar el dominio del inglés como lengua extranjera. Posteriormente, se detallan los lineamientos para el diseño de materiales educativos para la enseñanza de una lengua extranjera, propuestos por Tomlinson [3] y se señalan los parámetros del diseño instruccional ADDIE para la creación de aplicaciones móviles en la enseñanza del inglés como lengua extranjera. Finalmente, se exponen algunas conclusiones y reflexiones.

\section{Las TIC en el aprendizaje de una segunda lengua}

El plan educativo de aprendizaje (PEA) de una lengua extranjera en el aula de clase exige el uso de diferentes recursos convencionales, así como de herramientas modernas. Actualmente, en el mercado global existe un gran número de recursos de la Web 2.0 que impulsa a los docentes de lenguas extranjeras a capacitarse plenamente en su uso y a buscar un mejor entendimiento de la lengua meta. En este sentido, la práctica de un segundo idioma ocurre dentro y fuera del aula de clase, en la que se han de utilizar diversas herramientas audiovisuales. Sin embargo, dependiendo del centro educativo, en ocasiones estas herramientas no están disponibles para la profundización del idioma extranjero, sino que, en clases presenciales, el cuerpo docente emplea una gran cantidad de estrategias basadas en los modelos metodológicos de enseñanza tradicional, teniendo en cuenta las características e intereses de los estudiantes para suplir sus necesidades lingüísticas.

Es necesario, entonces, tanto emplear recursos de la enseñanza tradicional, como incorporar las tecnologías en el aula, para obtener mejores resultados académicos. Al utilizar las nuevas tecnologías en el salón de clase, los estudiantes y el cuerpo docente tienen la oportunidad de afianzar los conocimientos y construir un ambiente colaborativo. Así, se facilita el PEA de un idioma extranjero, al proveer un mejor ambiente de aprendizaje, puesto que los estudiantes se sienten más motivados a participar e interactuar con otros cuando emplean un objeto virtual de aprendizaje que conocen y utilizan diariamente. De este modo, se hace posible para los alumnos profundizar en los aspectos lingüísticos de un idioma extranjero interactuando con diversos recursos de la Web 2.0 y se facilita al cuerpo docente reorganizar sus prácticas, utilizando una flexibilización curricular en la cual se incorpora el uso de nuevas tecnologías, en especial las aplicaciones móviles. Esto, en todo caso, supone un potencial dinámico y didáctico para afianzar nuevos modelos metodológicos en el PEA de un idioma extranjero.

Las actividades didácticas destinadas a los estudiantes deben ser planeadas, pensadas y contextualizadas para que fortalezcan las habilidades lingüísticas en el idioma que se adquiere como segunda lengua. Su relevancia radica en su potencial para dar soluciones a las necesidades de las personas, ofreciendo ejemplos, ejercicios, recomendaciones y dinámicas de retroalimentación. Por tal motivo, se debe capacitar a los profesores de las escuelas, colegios y universidades en diversas competencias que puedan desarrollar en sus salones de clase en el uso de las aplicaciones móviles como medio tecnológico para transmitir información sobre un idioma extranjero. También es indispensable que las instituciones tengan la oportunidad de adquirir buenos dispositivos tecnológicos, que motiven al estudiante en el modelo pedagógico Learning by doing (aprender haciendo). Colaborando con los profesores, ayudándolos a innovar sus metodologías y creando materiales interactivos ajustados a sus necesidades, el estudiante se convierte en una persona productiva del PEA en el que se encuentra inmerso.

Las TIC incorporadas al aprendizaje de una segunda lengua permiten profundizar en el intercambio y colaboración instantánea con los compañeros, enriqueciendo mutuamente sus procesos. No se debe olvidar que, en este modelo, el alumno es el responsable de su propio aprendizaje y que necesita, por tanto, desarrollar autonomía, creatividad y capacidad de convertirse en el gestor de su tiempo para las actividades pautadas. Además, para los estudiantes virtuales, que cuentan con la posibilidad de trabajar a 
su propio ritmo, este modelo permite perfeccionar cada competencia lingüística ${ }^{3}$ a través de la diversidad de recursos, que pueden ser revisados en cualquier momento que se requiera.

\section{Diseño metodológico}

\subsection{Diseño tecnopedagógico}

El diseño tecnopedagógico es considerado como el conjunto de teorías, actividades y lineamientos, realizados por el cuerpo docente y centrados y contextualizados en el alumno, que tienen en cuenta sus intereses, características y necesidades de aprendizaje. En la Figura 1, tomada de Coomey y Stepheson, en Stephenson y Sangrà [4], se establecen cuatro cuadrantes teniendo en cuenta diversos modelos pedagógicos que varían de acuerdo con el tipo de actividades y tareas, y con el contenido y las funciones que ejercen los docentes y el alumnado.

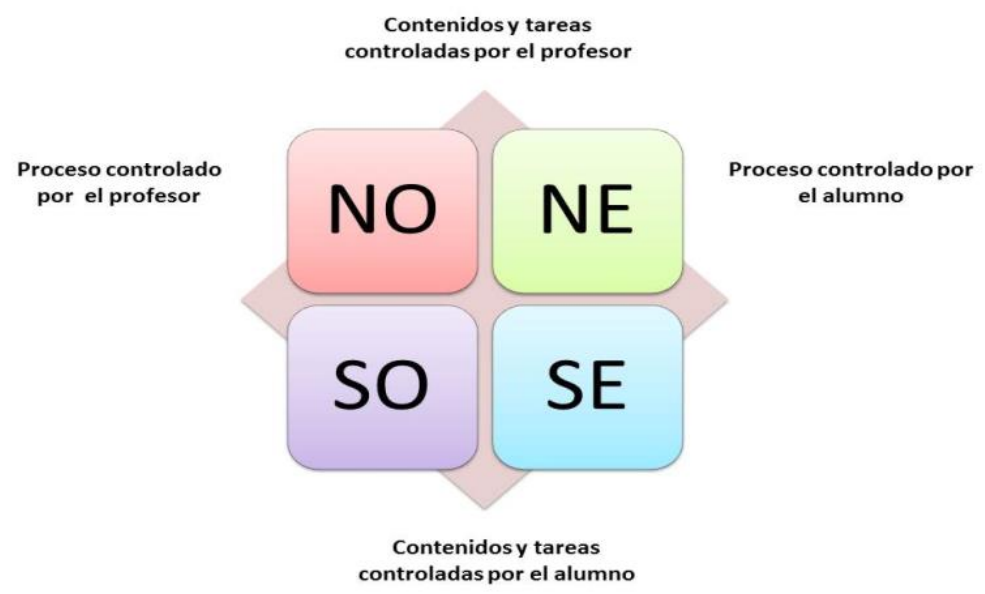

Figura 1. Cuadrícula de paradigmas pedagógicos de Coomey y Stepheson en Stephenson y Sangrà [4].

En la Tabla 1, se pueden observar las características del cuadrante noroeste (NO) relacionadas con los roles docente y alumno. Se puede colegir que el docente es quien establece las actividades por realizar, maneja el tiempo de entrega de los trabajos y contenidos desarrollados, mientras que el estudiante desempeña un rol pasivo en su proceso de aprendizaje, al limitarse a la recepción de información.

Tabla 1. Cuadrante noroeste de Coomey y Stephenson en Stephenson y Sangrà [4].

\begin{tabular}{|l|l|}
\hline \multicolumn{2}{|c|}{ Características del cuadrante NO } \\
\hline \multirow{4}{*}{ Diálogo } & $\begin{array}{l}\text { El profesor define/controla el diálogo y la interacción en línea. } \\
\text { El estudiante responde a las preguntas y minitareas del profesor. } \\
\text { El diálogo con los compañeros se especifica como parte de una tarea. } \\
\text { La concentración en el diálogo normalmente está orientada a la tarea de resolver un } \\
\text { problema. }\end{array}$ \\
\hline \multirow{3}{*}{ Implicación } & $\begin{array}{l}\text { Poco o ningún margen para que el alumno tenga influencia sobre el contenido. } \\
\text { La actividad se define y se relaciona estrictamente a una tarea preestablecida. El } \\
\text { sitio se estructura para conducir al alumno directamente a la información específica. } \\
\text { Los estudiantes pueden acceder a la información desde un sitio web antes, durante o } \\
\text { después de las clases. }\end{array}$ \\
\hline \multirow{2}{*}{ Apoyo } & $\begin{array}{l}\text { Se supone que proviene solamente del profesor a través de correo electrónico o } \\
\text { llamadas telefónicas o reuniones presenciales que están programadas. }\end{array}$ \\
\hline
\end{tabular}

${ }^{3}$ Las competencias lingüísticas evaluadas en una lengua extranjera son: escucha, habla, escritura y lectura. 


\begin{tabular}{|l|l|}
\hline & El feedback principal procede del formador. \\
\hline \multirow{3}{*}{ Control } & El control del alumno se limita a la respuesta a las tareas. \\
& Algún control sobre la secuenciación y el nivel de compromiso. \\
& El profesor controla el material de lectura, el contenido que hay que aprender, las \\
& fechas límite de entrega. \\
\hline Función del profesor & Formador. \\
\hline
\end{tabular}

Por otro lado, en la Tabla 2 se observan las características del cuadrante noreste propuesto por Coomey y Stepheson en Stephenson y Sangrà [4], quienes establecen algunos cambios en los roles del docente y estudiantado en comparación con el cuadrante noroeste. En este sentido, aunque el docente es quien controla el contenido y las tareas en el proceso de aprendizaje, el estudiante es la persona quien controla el proceso. Es decir, los alumnos gestionan cuándo y dónde aprenden, hay más interacción entre los compañeros y los estilos de aprendizaje se desarrollan más.

Tabla 2. Cuadrante noreste de Coomey y Stephenson en Stephenson y Sangrà [4].

\begin{tabular}{|c|c|}
\hline \multicolumn{2}{|c|}{ Características del cuadrante $\mathrm{NE}$} \\
\hline Diálogo & $\begin{array}{l}\text { El profesor establece las responsabilidades y los procedimientos generales, pero no } \\
\text { la participación, el contenido ni el uso. } \\
\text { El alcance se limita a la tarea, pero los sistemas y protocolos dan apoyo al diálogo } \\
\text { gestionado por el estudiante con otros estudiante, compañeros y expertos. }\end{array}$ \\
\hline Implicación & $\begin{array}{l}\text { Grupos autogestionados centrados en tareas. } \\
\text { Los grupos pueden ser autoseleccionados y/o automoderados. } \\
\text { El alumno es capaz de relacionar o adaptar tareas a sus propias circunstancias y } \\
\text { aspiraciones. }\end{array}$ \\
\hline Apoyo & $\begin{array}{l}\text { El tutor aconseja sobre la naturaleza de la tarea, las metas de aprendizaje, etc. } \\
\text { Principalmente, contacto por correo electrónico o grupos de debate moderados por el } \\
\text { tutor. } \\
\text { Los estudiantes proporcionan feedback a los miembros de su propio grupo y a otros. }\end{array}$ \\
\hline Control & $\begin{array}{l}\text { El control de la tarea depende del alumno. } \\
\text { Énfasis en vínculos navegables a una amplia variedad de fuentes. } \\
\text { Uso de recursos fuera del programa. } \\
\text { Amplia elección del alumno respecto a las actividades, el contenido y los resultados } \\
\text { de aprendizaje. }\end{array}$ \\
\hline Función del profesor & Entrenador. \\
\hline
\end{tabular}

En la Tabla 3 se encuentra información sobre las características del cuadrante sudoeste relacionadas con el rol del docente y del aprendiz. El docente es un monitor, investigador, reflexivo solucionador de problemas e investigador de la acción; es un diseñador tecnopedagógico, un guía orientador y facilitador del proceso de los estudiantes, tal como lo sugieren Stephenson y Coomey en Stephenson y Sangrà [4]. Sus funciones se enfocan en preparar y dar la clase magistral, ofrecer bibliografías, hacer exposiciones, trabajos en grupo en el aula y estudios de caso. En este sentido, se obtiene un proceso de aprendizaje de acuerdo con el compromiso del estudiante por seguir investigando y la evaluación se convierte en algo riguroso. Asimismo, el aprendiz ejerce un rol participativo y activo en todo el proceso de aprendizaje; es reflexivo, crítico y pensante sobre las diferentes actividades que desarrolla, con el objetivo de poder interactuar en diversos entornos virtuales de aprendizaje. Es además capaz de manejar su propio entorno, en donde tiene la posibilidad de escoger varios recursos tecnológicos que sean adecuados para el área a profundizar. Sin embargo, la creatividad y falta de materiales multimedia conduce, en algunas ocasiones, a que el estudiante sea pasivo frente a su aprendizaje.

Tabla 3. Cuadrante sudoeste de Coomey y Stephenson en Stephenson y Sangrà [4].

Características del cuadrante SO

\begin{tabular}{|l|l|}
\hline Diálogo & Una combinación de estilos de diálogo que se encuentran en el NO, durante el \\
\hline
\end{tabular} 


\begin{tabular}{|c|c|}
\hline & $\begin{array}{l}\text { segmento dirigido por el formador del curso y de SE durante el segmento del curso } \\
\text { gestionado por el alumno. } \\
\text { Podría ser controlado por el profesor y centrarse en la dirección y el objetivo } \\
\text { generales del estudio. } \\
\text { Uso de diálogo asincrónico pero con funciones establecidas por el instructor para los } \\
\text { estudiantes, haciendo que los estudiantes participen como líderes o bien ofreciendo } \\
\text { respuestas en debates o pidiendo a los estudiantes que clasifiquen sus respuestas. }\end{array}$ \\
\hline Implicación & $\begin{array}{l}\text { Podría comenzar como una actividad en solitario en la que el estudiante aprenda } \\
\text { reglas / conceptos / teorías a partir de textos en línea y posiblemente clases } \\
\text { tradicionales. } \\
\text { El texto puede estar en línea pero también hay ubicaciones para que los estudiantes } \\
\text { escriban y sitúen sus "descubrimientos", (vínculos, datos y contenido). Una vez que } \\
\text { los estudiantes dominan "lo básico", crean algo nuevo por sí mismos. } \\
\text { Las actividades de grupo se limitan principalmente al grupo del curso. }\end{array}$ \\
\hline Apoyo & $\begin{array}{l}\text { El apoyo del tutor podría ser en línea o, en ocasiones, presencial. } \\
\text { Gama de apoyo: el feedback tradicional en la primera fase del curso (cuadrante NO)/ } \\
\text { el formador actúa como facilitador, ofrece sugerencias pero no respuestas durante la } \\
\text { fase de "descubrimiento" del curso (cuadrante SE). }\end{array}$ \\
\hline Control & $\begin{array}{l}\text { El estudiante controla las metas de aprendizaje específicas dentro de las metas } \\
\text { generales. } \\
\text { Gestión de actividades de descubrimiento desestructuradas dentro de parámetros } \\
\text { concretos. } \\
\text { Libre de establecer metas personales propias dentro de la actividad generalizada. }\end{array}$ \\
\hline Función del profesor & Guía. \\
\hline
\end{tabular}

Tabla 4. Cuadrante sudeste de Coomey y Stephenson en Stephenson y Sangrà [4].

\begin{tabular}{|l|l|}
\hline \multicolumn{2}{|c|}{ Características del cuadrante SE } \\
\hline \multirow{2}{*}{ Diálogo } & $\begin{array}{l}\text { Autodirigido o dirigido en colaboración con los compañeros del grupo. } \\
\text { Amplia elección del alumno respecto a la selección de grupos de debate, desde } \\
\text { compañeros a grupos de interés especializados "públicos". } \\
\text { Diálogo asincrónico con otros especialistas. } \\
\text { Fuente externa de ayuda de especialista. }\end{array}$ \\
\hline \multirow{5}{*}{ Implicación } & $\begin{array}{l}\text { Implicación total en la actividad de aprendizaje. } \\
\text { Podría trabajar solo o en equipo. } \\
\text { El alumno relaciona el aprendizaje a sus propias necesidades personales, } \\
\text { vocacionales o académicas. }\end{array}$ \\
\hline Apoyo & $\begin{array}{l}\text { El alumno inicia y controla el contacto con el supervisor facilitado por el sistema. } \\
\text { El profesor está en su segundo plano desde el que ofrece asesoramiento sobre } \\
\text { procedimientos y recursos. } \\
\text { Se busca el feedback de diversas fuentes y personas expertas. } \\
\text { La estructura y el diseño del aprendizaje en línea proporcionan un marco de apoyo } \\
\text { dentro del cual el alumno tiene una elección considerable. }\end{array}$ \\
\hline Control & $\begin{array}{l}\text { El alumno determina las metas y los resultados. } \\
\text { El alumno controla el progreso. }\end{array}$ \\
\hline Función del profesor & Facilitador. \\
\hline
\end{tabular}




\subsection{Diseño instruccional ADDIE}

El diseño tecnopedagógico es un instrumento útil en el proceso enseñanza - aprendizaje, pues permite a los estudiantes fortalecer su nivel de dominio de la lengua y mejorar sus competencias orales y escritas del inglés, por medio de ejercicios y actividades que se adecúan a sus intereses. Un buen diseño tecnopedagógico facilita la adquisición de destrezas ortográficas y de gramática inglesa y la adecuada utilización de la forma de las palabras y su uso en el orden correcto. A través del desarrollo de las diferentes actividades, los estudiantes adquieren mayor vocabulario. La importancia del diseño tecnopedagógico radica en ser la mejor forma de utilizar el diseño instruccional interactivo ADDIE, el cual es un proceso de aprendizaje cuyos resultados en la evaluación formativa de cada etapa pueden retroalimentar las etapas restantes y llevar al diseñador instruccional de regreso a cualquiera de las fases previas. De esta manera, el producto final de una fase se constituye en el producto inicial de la siguiente fase. Este modelo facilita evaluar las necesidades de los estudiantes, analizar las tareas y utilizar los recursos disponibles para el desarrollo de una acción educativa, tales como foros, tablones de anuncios, ejercicios, audios e imágenes. También, permite realizar el manual del usuario y las actividades, revisar el material existente y trabajar con los productores para desarrollar los medios. La Figura 2 ilustra esquemáticamente el modelo ADDIE y de ella se pueden colegir las siguientes observaciones.

\begin{tabular}{|c|c|}
\hline \multirow{5}{*}{$\begin{array}{l}\text { El modelo } \\
\text { ADDIE es un } \\
\text { proceso de diseño } \\
\text { instruccional } \\
\text { interactivo, en } \\
\text { donde los } \\
\text { resultados de la } \\
\text { evaluación } \\
\text { formativa de cada } \\
\text { fase pueden } \\
\text { conducir al } \\
\text { diseñador } \\
\text { instruccional de } \\
\text { regreso a } \\
\text { cualquiera de las } \\
\text { fases previas. El } \\
\text { producto final de } \\
\text { una fase es el } \\
\text { producto de inicio } \\
\text { de la siguiente } \\
\text { fase. }\end{array}$} & $\begin{array}{l}\text { Análisis: Analizar el alumnado, el contenido y el entorno cuyo resultado } \\
\text { será la descripción de una situación y sus necesidades formativas. }\end{array}$ \\
\hline & $\begin{array}{l}\text { Diseño: Desarrollo de un programa de curso deteniéndose especialmente en } \\
\text { el enfoque pedagógico y en el modo de secuenciar y organizar el contenido. }\end{array}$ \\
\hline & $\begin{array}{l}\text { Desarrollo: La creación real (producción) de los contenidos y materiales de } \\
\text { aprendizaje basados en la fase de diseño. }\end{array}$ \\
\hline & $\begin{array}{l}\text { Implementación: Ejecución y puesta en práctica de la acción formativa con } \\
\text { la participación de los alumnos. }\end{array}$ \\
\hline & $\begin{array}{l}\text { Evaluación: Esta fase consiste en llevar a cabo la evaluación formativa de } \\
\text { cada una de las etapas del proceso ADDIE y la evaluación sumativa a través } \\
\text { de pruebas especificas para analizar los resultados de la acción formativa. }\end{array}$ \\
\hline
\end{tabular}

Figura 2. Modelo instruccional ADDIE. Fuente: adaptación de entornos virtuales de formación, Universidad de Valencia, España.

Análisis: el docente de lenguas extranjeras debe realizar un análisis de necesidades de sus usuarios para realizar el diseño de actividades acordes a sus intereses y necesidades. Se debe:

- Definir el perfil de la población objetivo.

- Definir los objetivos que deben desarrollar los usuarios.

- Identificar el contexto de aprendizaje de la acción formativa.

- Identificar las competencias de los usuarios.

- Identificar los problemas y estilos de aprendizaje de los usuarios.

- Identificar los recursos tecnológicos dispuestos por la institución educativa.

- Definir la estructura para llevar a cabo la misión de la empresa.

- Definir la plataforma tecnológica empleada en la acción formativa.

- Definir los recursos utilizados en las temáticas planteadas. 
Diseño: en esta fase, el docente de una segunda lengua ejerce el rol de diseñador instruccional y curricular y se encarga de definir los objetivos del aprendizaje y dar una visión clara de las estructuras del curso, temáticas y dinámicas de aprendizaje, entre otros, para que el alumnado alcance los objetivos. En este sentido, se debe:

- Definir el modelo pedagógico, enfoque didáctico y teorías de aprendizaje en la acción formativa.

- Definir los cuadrantes pedagógicos propuestos por Coomey y Stepheson, en Stephenson y Sangrà [4].

- Identificar el modelo instruccional en la acción formativa.

- Definir la secuencia de contenido en la acción formativa.

- Diseñar las actividades tecnopedagógicas en la acción formativa.

- Identificar los recursos tecnológicos para el desarrollo de la acción formativa.

- Producir y ejecutar el contenido en la acción formativa.

- Usar y aplicar los recursos tecnológicos en la acción formativa.

- Evaluar el uso de los recursos tecnológicos en la acción formativa.

- Analizar el impacto de la acción formativa.

Desarrollo: en esta sección, el docente de lenguas extranjeras debe proceder con la organización de los elementos para llevar a cabo los cursos, que se dictarán de manera didáctica y constructiva para profundizar en el aprendizaje significativo.

Implementación: el docente se centra en la implementación total del curso teniendo en cuenta los pasos mencionados anteriormente.

Evaluación: se realizará de manera continua durante el curso y al final del mismo con la implementación. Se evalúa la presentación del curso, en donde se evidencia el aprendizaje adquirido en las temáticas planteadas, los materiales de formación, las formas de interacción y la apropiación de la experiencia de la acción formativa. En este sentido, se puede realizar una evaluación formativa y sumativa de la acción de aprendizaje.

Es importante señalar que las Apps y Mobile Learning son un medio de gran utilidad para el refuerzo de una lengua extranjera, ya que facilitan la realización de diversas actividades y el desarrollo de ejercicios planteados. El empleo adecuado de la interfaz de la aplicación educativa permite obtener experiencia en la estructuración de textos, haciendo que la misma sea coherente para los lectores. El usuario tiene, igualmente, la posibilidad de discriminar los diferentes sonidos y reconocer los patrones de ritmo y entonación de la lengua inglesa. Las actividades didácticas propuestas en el diseño tecnopedagógico están centradas en el alumno; su pertinencia y relevancia radican en que guían y ayudan en forma real a los estudiantes, quienes tienen la capacidad de poner a prueba sus competencias en el manejo de los diferentes recursos de lengua para la realización de ejercicios, cualquiera sea el módulo que ellos consideren que deban reforzar. En efecto, un buen y estructurado diseño tecnopedagógico se convierte en una potente herramienta que facilita el proceso enseñanza - aprendizaje de un idioma extranjero.

\subsection{Modelo pedagógico Learning by doing}

De acuerdo con Stepheson y Sangrà [4], en el modelo pedagógico learning by doing, que significa "aprender haciendo" o "aprender por la práctica", el estudiante juega un rol activo, ya que tiene la posibilidad de intercambiar experiencias con otras personas por medio de actividades de las que puede inferir el conocimiento adquirido. El modelo pedagógico se debe centrar en el alumno, de modo que él sea el centro de atención de su propio proceso de aprendizaje, utilizando la tecnología como un medio de enseñanza; es el propio alumno quien descubre el conocimiento mediante la práctica. Por esta razón, es fundamental que los estudiantes sean instruidos en esta área, con el objetivo de utilizar estas herramientas efectiva y adecuadamente en sus labores educativas. Por lo tanto, en el mundo educativo, las TIC ${ }^{4}$ son empleadas en diversos ámbitos didácticos con el propósito de afianzar más los conocimientos adquiridos, así como fomentar y desarrollar las habilidades cognitivas para que la tarea establecida pueda ser lograda

\footnotetext{
4 Acrónimo utilizado para referirse a las Tecnologías de Información y Comunicación.
} 
a cabalidad. Por consiguiente, es necesario tener en cuenta los diversos estilos de aprendizaje de los alumnos, que pueden ser, por ejemplo, activos, reflexivos, teóricos y pragmáticos, para lograr un mayor progreso en el reforzamiento de una segunda lengua.

\subsection{Enfoque de la acción}

El “enfoque de la acción”, propuesto en el año 2000 por el Marco Común Europeo de Referencia para las lenguas extranjeras, es un método complementario a la teoría comunicativa, en el que el estudiante desempeña un importante papel social, encara dificultades y resuelve problemas reales en contextos específicos. En este enfoque metodológico, los estudiantes deben crear productos finales en forma de proyectos; en ellos, deben emplear un lenguaje auténtico de acuerdo con sus necesidades y con los aspectos socio-culturales del aprendizaje de una lengua extranjera. En el modelo propuesto, el estudiante realiza un proceso de aprendizaje en tres etapas en el marco del "ciclo de la tarea", así: pre-tarea, tarea y post-tarea. La "pre-tarea" desarrolla un sentido crítico en la selección tanto de contenido como de recursos. La "tarea" facilita al alumno la comprensión y aprendizaje de los diferentes aspectos de cada tipo de texto, teniendo en cuenta la función del objeto del mismo y la audiencia a la que va dirigido. Por último, la "post-tarea", estimula la capacidad de asimilar la crítica, propiciar la autocrítica y adquirir la competencia de aceptar e implementar la retroalimentación que pueda ser recibida, para mejorar el producto creado.

\section{Trabajos relacionados}

\subsection{La influencia del diseño tecnopedagógico en los procesos de refuerzo del inglés como lengua extranjera para la aprobación de un examen internacional}

En líneas previas se ha planteado la importancia del uso de las TIC en la formación profesional y como herramienta para la apropiación y/o el reforzamiento de conocimientos. En virtud de tales tecnologías, se han podido crear ambientes de interacción social y de formación de talento humano. En el campo del aprendizaje de segundas lenguas, las TIC han permitido a las personas, por una parte, interactuar, vivenciar y ganar experiencias de aprendizaje y, por otra, apropiarse y crear nuevos conocimientos para transformar el ambiente social y laboral en que viven.

La evaluación del rol de las TIC como herramientas facilitadoras de aprendizaje conllevó revisar las distintas maneras en que las personas aprenden. Una respuesta a tales miradas consiste en el cambio de perspectiva que ha encontrado la investigación en asuntos educativos, al pasar de una investigación centrada en la educación presencial a otras modalidades formativas, incluida la educación a distancia semipresencial (inicialmente enfocada en la enseñanza, mas no en el aprendizaje) y, más recientemente, la educación netamente virtual (vista desde el aprendizaje).

El convenio de asociación E-Learning 2.0 Colombia [5], en su documento Propuesta de metodología para transformar programas presenciales a virtuales o E-learning, plantea las dimensiones para el desarrollo de programas de educación virtual, e-learning, on-line learning, educación distribuida o educación a distancia de tercera generación y otras formas instruccionales. Estas están orientadas no solo a reducir o eliminar las barreras de tiempo y distancia, sino a transformar los modelos de educación frontal y de institución educativa tradicional, mediante el uso racional y adecuado de herramientas tecnológicas. El mismo estudio, al reconocer la complejidad del aprendizaje, propone los lineamientos pedagógicos que podrían fundamentar la reforma de los programas educativos tradicionales para ser ofertados en la modalidad virtual. Tal dimensión ha dinamizado a las instituciones educativas en la dirección de fortalecer sus entornos de aprendizaje y sus logísticas tecnológicas, creando ambientes de acceso al conocimiento que incluyen los métodos presenciales de la educación tradicional y la implementación de plataformas tecnológicas de interacción y ambientes virtuales de aprendizaje, definidos para la educación a distancia, en el contexto de la educación virtual. 
El empleo de escenarios de ambientes virtuales de aprendizaje fue adoptado inicialmente en las instituciones de educación superior para fortalecer los procesos de la formación presencial y a distancia. En la actualidad, es el soporte de algunas instituciones de educación superior para ofertar sus programas de pregrado y postgrados en modalidad virtual. En el marco de la globalización, el uso de las TIC y de la educación virtual ha impactado la sociedad global en términos económicos, tecnológicos, sociales y culturales. Uno de los aspectos de mayor relevancia en la globalización se refiere a la comunicación y a la necesidad de forzar la enseñanza del bilingüismo. El Marco Común Europeo de Referencia es quizás el referente más conocido y empleado como estándar internacional para medir el nivel de comprensión y expresión orales y escritas en una segunda lengua. A tono con esta dinámica mundial, el Ministerio de Educación Nacional de Colombia [6] propuso la implementación del Programa Nacional de Bilingüismo con el propósito de que todos los ciudadanos fuesen capaces de comunicarse en inglés, con estándares internacionalmente comparables, de tal forma que se pudiese insertar al país en los procesos de comunicación universal, en la economía global y en la apertura cultural, con estándares internacionalmente comparables.

Sin embargo, es preciso señalar que, si bien algunos autores (Schwienhorst, 2012 [7]; Slater et al., 2006 [8]; Visser, 1999 [9]; entre otros), sostienen que el aprendizaje apoyado en el computador interfiere con el desarrollo de habilidades socio pragmáticas, de interacción y de transacción, Driessen y sus colegas mencionan que el empleo de las TIC en el aprendizaje formal de una segunda lengua tiene un enorme potencial por explotar [10]).

Se entiende que el empleo de una nueva herramienta tecnológica supone siempre oportunidades y obstáculos, ventajas y desventajas. Por tal motivo, existe la necesidad de evaluar los nuevos insumos y medios tecnológicos aportados por la ciencia. Esta tarea puede llevarse a cabo a través de un análisis matricial DOFA (debilidades, oportunidades, fortalezas y amenazas), con el fin de alinerse en la dirección proactiva de los nuevos aprendizajes, en la búsqueda de romper viejos esquemas y eliminar barreras que dificulten una masificación de la educación de calidad. El auge y masificación de la información y la comunicación han puesto a la educación virtual en el primer plano de los sistemas educativos, exigiendo más innovación en los procesos de enseñanza - aprendizaje, apoyados en herramientas tecnológicas.

Miranda, Morais y Dias [11] presentan algunas reflexiones sobre estrategias pedagógicas basadas en creaciones tecnológicas, que pueden ser incorporadas en los modelos pedagógicos para posibilitar la educación en línea. Los recursos tecnológicos y de tipo contextual son muy relevantes para el diseño de un ambiente virtual de aprendizaje. Para tener un impacto positivo en los procesos de aprendizaje y efuerzo de una lengua extranjera, por medio de la internet, es necesario que el usuario conozca, comprenda y se familiarice con el uso de las plataformas virtuales en la enseñanza de lenguas extranjeras que hayan sido diseñadas para posibilitar la comunicación pedagógica entre los participantes en un proceso educativo, según lo plantean Fernández y Pampillón [12]. Este propósito es una tarea compleja, debido al lenguaje técnico empleado y a la contradicción hallada, según palabras de los autores, mencionados: "entre la potencialidad teóricamente predicha para el e-learning a principios del año 2000, y los pobres resultados obtenidos, especialmente en términos económicos, en los siguientes años [12]";

Fernández y Pampillón [12] hacen énfasis en las plataformas educativas generales, señalando que:

\begin{abstract}
Estas no tienen como objetivo el aprendizaje de una materia concreta o hacia la adquisición de una competencia en particular o a la realización de una función específica [...]. En este campo se encuentran distintas plataformas, tales como: Blackboard y Moodle, y son las que se encargan de mejorar la eficacia y eficiencia académica, especializándose en determinadas áreas de conocimiento [12].
\end{abstract}

Para el caso del nuevo modelo propuesto, el desarrollo de la interfaz de la aplicación debe estar orientado hacia un aprendizaje tecno-socio constructivista que dé prevalencia a la autonomía y al aprendizaje autónomo de los estudiantes. La internet es un potente medio en el campo de la enseñanza de lenguas extranjeras, tal como lo sugiere Tomé [13]. No obstante, para este autor, el rol de la lengua oral es muy limitado en internet, porque a su juicio aún se requieren equipos muy potentes para cargar archivos de sonido, al tiempo que apenas se están resolviendo los problemas técnicos planteados por los sistemas multimedia para que sean eficaces y ágiles dentro del entorno de internet.

De esta manera, las restricciones tecnológicas actuales imposibilitan, por el momento, la presencia de documentos de audio y ciñe el aporte de la internet a la presencia masiva de documentos escritos o imágenes. Lo anterior sugiere que la enseñanza de lengua extranjera en internet se mantiene dentro de los 
límites conocidos de las metodologías tradicionales, a la espera de que los sistemas multimedia puedan entrar a formar parte efectiva de la red.

En materia de ambientes web instruccionales para lenguas, Pedraza y Berdugo [14] describieron algunos sistemas de enseñanza en línea y aplicaciones informáticas e investigaciones sobre herramientas web útiles para apoyar procesos de enseñanza - aprendizaje, destacando aquellas enfocadas a la enseñanza de lenguas. Asimismo, tales autores revisaron las bases del diseño instruccional que orientan las aplicaciones web educativas y presentaron los principios cognitivos y sociocognitivos del aprendizaje y la conceptualización del diseño en el marco teórico de dichos principios. Concluyeron que, en el diseño de ambientes virtuales para la enseñanza y aprendizaje de lenguas extranjeras, se deben tener en cuenta los siguientes factores:

En lo que concierne al diseño de ambientes hipermediales para la enseñanza y aprendizaje de lenguas, el reto radica también en tender puentes entre la información educativa, y la didáctica de lenguas, de manera que se articulen, de forma adecuada, las perspectivas teóricas y metodológicas del DI en general con las especificidades de la enseñanza y aprendizaje de los idiomas [14].

También, Argüelles y García [15], en sus investigaciones, hallaron que el aprendizaje tiene algunos beneficios a partir de los avances tecnológicos. Señalan que estos amplían los horizontes del conocimiento, pues, al integrar la información con diferentes medios multimedia e hipermedia, realmente los estudiantes interactúan con los conceptos, ya que:

\begin{abstract}
La persona se enfrenta en diálogo abierto con el autor, analiza y sintetiza la información, la reordena y construye significados, de acuerdo con sus conocimientos previos y con sus experiencias y vivencias; confirma o modifica su visión del mundo, acorde con su percepción del conocimiento. Es decir, aprende a aprender en forma autónoma [15].
\end{abstract}

Por consiguiente, es de vital importancia seguir profundizando en la temática discutida, con el objetivo de proponer herramientas tecnológicas que otorguen a los usuarios la posibilidad de mejorar sus procesos de aprendizaje de un segundo idioma.

El Informe Mundial de la Educación de la UNESCO [16] hace explícito el reconocimiento de las TIC, al establecer como postulado que:
Hay que utilizar plenamente el potencial de las nuevas tecnologías de la información y la comunicación para la renovación de la educación superior, mediante la ampliación y diversificación de la transmisión del saber, y poniendo los conocimientos y la información a disposición de un público más amplio [16].

Por su parte, el mismo informe menciona que "es importante señalar que las nuevas tecnologías brindan posibilidades de renovar el contenido de los cursos y los métodos pedagógicos, y de ampliar el acceso a la educación superior [16]". Esa misma Organización expresa en el Informe Mundial sobre el Aprendizaje y la Educación de Adultos [17] que:
Las nuevas tecnologías de la información y la comunicación (TIC) intensifican la tasa de intercambio de información. También permiten que los usuarios participen activamente en redes virtuales que se pueden movilizar fácilmente para modelar la opinión pública [17].

En Colombia, solo hasta comienzos del presente milenio se formula la Agenda de Conectividad: El Salto a Internet (CONPES 3072), como política de Estado a largo plazo, la cual, de conformidad con la Política Nacional de Ciencia y Tecnología 2000-2002, consistía en la articulación de un conjunto de estrategias para el aprovechamiento de las tecnologías de la información en el desarrollo económico, social y político del país (CONPES 3080). Tal agenda, coordinada inicialmente por el Programa Presidencial para el Desarrollo de las TIC, pasó a ser responsabilidad del Ministerio de Comunicaciones, al suprimirse las funciones presidenciales. Para Meza y Soler [18]:

\footnotetext{
La situación de Colombia a comienzos del año 2000, según el CONPES 3072, presentaba un rezago frente a la tendencia mundial en los aspectos relacionados con la infraestructura computacional y social (mide la capacidad que tienen los ciudadanos de aprovechar a las TIC). Esta disparidad se hacía menos notoria para el caso de la infraestructura de información (capacidad de recibir y enviar información en formatos de voz, texto y video), lo que hizo que se
} 
reconociera como un problema colectivo y cuya no intervención traería como una de sus consecuencias, el aumento de la brecha digital con respecto a los países desarrollados [18].

Acorde con la política de conectividad, el Estado colombiano, a través del MEN y MINTIC, ha venido fomentando y dando celeridad a la masificación del uso de las TIC y estimulando la creación de ambientes favorables para incentivar la apropiación de estas tecnologías. Esto con el propósito de alentar la aplicación de las mismas dentro de los organismos adscritos al Estado, la educación y el sector productivo, bajo la premisa de lograr mayores y mejores niveles de eficiencia y eficacia y poder acceder a nuevos mercados.

Las redes Mutis y Red de Ciencia, Educación y Tecnología de Colombia (CETCOL), la Red Nacional Universitaria, el Banco de Proyectos en diversas disciplinas del conocimiento y la conexión de las bibliotecas nacionales (Biblioteca Virtual) son ejemplos de los esfuerzos gubernamentales para posicionar las TIC en el territorio nacional (CONPES 3072) [19], tanto en el sector académico y el sector privado como en la sociedad en general. De conformidad con Meza. J. F, y Soler F. Ángela [18], el MEN, en el marco del Programa Nacional de Uso de Medios y Nuevas TIC, para el período 2007-2010, reformuló los componentes del mencionado programa, en:

\begin{abstract}
Uso y apropiación de medios y TIC (redes y comunidades virtuales), desarrollo profesional del recurso humano (formación de tutores para AVA), gestión de contenidos (Portal Educativo Colombia Aprende y Red Nacional de Bancos de Objetos de Aprendizaje) y gestión de infraestructura tecnológica (RENATA) [18].
\end{abstract}

Se destacan estrategias con influencia en la educación superior, tales como:

a) El Banco de Objetos Virtuales de Aprendizaje: creado en el año 2005, mediante concurso nacional dirigido a la educación superior y convocado por el MEN. En 2006 y 2007 el MEN financió y suscribió convenios con universidades para divulgar objetos informativos y de aprendizaje, generados en tales instituciones, concluyendo con la creación del banco de objetos de aprendizaje nacional, integrado por trece (13) instituciones, con más de 3.000 objetos catalogados [20].

b) Red Virtual de Tutores (RVT): es una estrategia dirigida a las IES, de carácter voluntario y abierto a nivel nacional e internacional, con el propósito de dar continuidad al proceso de capacitación ofertado por el MEN a docentes en el manejo de AVA y consolidar una red de comunidades virtuales de aprendizaje entre los tutores.

c) Red Nacional de Tecnología Avanzada (RENATA): es la red que conecta, comunica y propicia la colaboración entre la comunidad académica y científica de Colombia con la comunidad académica internacional y los centros de investigación más desarrollados del mundo (www.renata.edu.co).

Ciertamente, en lo relativo a la enseñanza de idiomas extranjeros, el auge de las comunicaciones y el apoyo de las TIC han facilitado la formulación de modelos pedagógicos de reforzamiento del aprendizaje de un idioma extranjero, aplicados, algunos, en el aula tradicional; otros, en el medio laboral o de residencia del aprendiz; y algunos, en ambientes mixtos.

La revisión documental, a partir de bases de datos, muestra trabajos investigativos que se vinculan con el desarrollo individual de tópicos pedagógicos, como estrategias de aprendizaje de lectoescritura basadas en modelos instruccionales SRSD, el diseño tecnopedagógico en la enseñanza de lengua extranjera, las TIC en ambientes educativos, el enfoque de la acción, entre otros. Usualmente, se menciona el aula tradicional para hacer referencia al ambiente de aprendizaje. No obstante, la creciente dependencia tecnológica, propia de la sociedad actual, ha forzado la diferenciación entre espacio físico, por una parte, y ambiente en el que se materializan los procesos de enseñanza - aprendizaje, por la otra.

Actualmente, la implementación de las TIC en la actividad instruccional formativa ha obligado a las instituciones educativas a renovar sus ambientes de aprendizaje convencionales, a eliminar las barreras espacio temporales existentes entre ambientes formativos, a cambiar metodologías y estrategias de aprendizajes y a reorientar la función docente. De acuerdo con la UNESCO:

La nueva tecnología de la información no hace que los docentes dejen de ser indispensables, sino que modifica su papel en relación con el proceso de aprendizaje, y que el diálogo permanente que transforma la información en conocimiento y comprensión pasa a ser fundamental [16] y con ello ha potenciado la educación virtual. 
La orientación pedagógica asistida por computador (CALL) inició en las décadas de los cincuenta y setenta del siglo pasado y su empleo se extiende hasta nuestros días, en el desarrollo de ambientes virtuales de aprendizaje, masificados a través de internet. Sin embargo, en América Latina, tales enfoques debieron esperar hasta los años noventa, con la popularización del uso de los computadores. La clasificación "CALL integrador" fue ideada con el objetivo de aunar tecnologías web 2.0 y los recursos multimedia, en la enseñanza de un idioma extranjero. En este sentido, se crearon programas con el propósito de proveer instrumentos de enseñanza a los profesores de lengua, tales como: Camsoft's Fun with Texts y The Authoring Suite. El aprendizaje de lenguas asistido por ordenador está centrado en tres clasificaciones:

- CALL conductista: década de los cincuenta, sesenta y setenta.

- CALL comunicativo: década de los setenta y ochenta.

- CALL integrador: década de los noventa en adelante.

De igual forma, Levy [21, 22] desarrolló una investigación sobre el aprendizaje de lenguas asistido por computador. Esta se orientó a encontrar aplicaciones para la enseñanza y aprendizaje de un idioma extranjero.

Del mismo modo, es necesario profundizar en los parámetros necesarios para el diseño de aplicaciones tecnológicas que faciliten el aprendizaje de lenguas extranjeras. De acuerdo con Mestre de Mogollón y sus coautores [23], este diseño de aplicaciones tecnológicas debe ser "usable" y "accesible" en términos de interacción entre el usuario y la aplicación móvil, utilizando diversos formatos de contenidos con el propósito de obtener llamativos estándares multimediales que atraigan la atención del usuario meta. Es necesario, también, emplear un diseño de la aplicación tecnológica adaptable a cualquier dispositivo móvil. Asimismo, se debe tener en cuenta que la interfaz de la aplicación tecnológica debe contener aspectos en relación con el tema que se desee tratar, así como "íconos asociados con configuración de la aplicación". También, se debe considerar la "disponibilidad" de información en cuanto a la prestación del servicio (24/7) y al uso de internet y banda ancha, para obtener una interacción eficaz y clara entre el usuario y el objeto de aprendizaje.

De igual forma, la "flexibilidad" del diseño de aplicaciones tecnológicas debe ser oportuna para los usuarios, orientada a la adaptación del diseño para suplir sus necesidades, teniendo en cuenta la estructura curricular para abordar el afianzamiento de una lengua extranjera. Además, este diseño debe ser "abierto", es decir, basado en software libre, para que todos los usuarios puedan acceder al mismo.

\subsection{Lineamientos para el diseño de materiales educativos propuestos por Tomlinson en la enseñanza de una lengua extranjera}

Con el vertiginoso crecimiento tecnológico ha evolucionado el diseño y aplicación de material educativo. Esto se comprueba, no solo desde la interacción con el material, sino en la dinámica operativa de las propias casas editoriales y en las técnicas aplicadas por los diseñadores. Incluso, puede decirse que la elaboración de material educativo se ha democratizado y, con ello, reducido la casi exclusividad que tenían las casas editoriales (Cambridge y Oxford, entre otras) para la creación de materiales de la enseñanza y aprendizaje de una lengua extranjera. De conformidad con los postulados de la UNESCO en materia de educación superior, las políticas colombianas de socialización y posicionamiento del uso de las TIC, lideradas por el MEN, han impulsado los desarrollos investigativos adelantados por las universidades y la copiosa producción intelectual divulgada en las bases de datos. Los temas principales se relacionan con la aplicación de las TIC en los procesos de enseñanza - aprendizaje y la creación de ambientes virtuales formativos.

Se colige, por tanto, que los docentes han incursionado en la dinámica de utilizar las herramientas computacionales como medio de enseñanza y han comprendido la importancia de elaborar sus propios diseños de materiales pedagógicos. Estos se transforman en herramientas guías para el uso de los estudiantes, tomando en cuenta sus contextos, necesidades e intereses. De acuerdo con Tomlinson [3], cuando un docente conoce las preferencias, intereses y opiniones de sus alumnos, puede utilizar con éxito los materiales didácticos para atraer la atención del estudiante. Este autor propone los siguientes lineamientos para lograr que un buen diseño de materiales educativos didácticos, factibles de utilizar en la 
enseñanza y/o profundización de una lengua extranjera, sea considerado adecuado a las necesidades de los estudiantes:

\begin{abstract}
Novedoso (inusuales tópicos, ilustraciones y actividades), variados (utilizar diferentes tipos de textos, emplear un gran número de varias voces de instructor en un $\mathrm{CD}$ ), presentación llamativa (emplear colores atractivos, una gran cantidad de espacio blando y fotografías), contenido adecuado (temas de interés al público destinado, temas que ofrezcan la posibilidad de aprender algo nuevo, historias interesantes, temas universales, referencias locales), actividades de desafio (tareas que estimulen a los estudiantes a pensar) [3].
\end{abstract}

\title{
5. Conclusiones y recomendaciones
}

Es innegable que la dinámica de la sociedad moderna tiene una fuerte dependencia tecnológica, puesto que ha facilitado la vida cotidiana, ha influenciado y constituido nuevas dimensiones culturales, modificado las costumbres y propuesto nuevas formas de aprendizaje. En el ámbito educativo, ha aportado a la construcción de nuevas modalidades de formación, ha abierto espacios para la educación virtual y ha eliminado las barreras de tiempo y espacio en tales procesos. Se ha mostrado cómo el auge y masificación de la información y la comunicación han puesto la educación virtual en un primer plano en los sistemas educativos, pues exigen a los estudiosos pedagógicos la tarea de innovar PEA, apoyados en herramientas tecnológicas de información.

Las TIC, especialmente la internet, desempeñan un papel vital en la enseñanza de una lengua extranjera y su masificación ha puesto al alcance de cualquier persona la posibilidad del bilingüismo. Los mejores materiales didácticos, por su versatilidad, familiaridad, eficacia y eficiencia, son aquéllos cuyos diseños han tenido en cuenta los diversos modos y estilos de aprendizaje de los estudiantes.

El diseño tecnopedagógico tiene no solo el objetivo de afianzar los conocimientos adquiridos en los estudiantes, sino el de desarrollar en ellos habilidades cognitivas y metacognitivas para la realización de tareas en su quehacer diario. La integración del diseño tecnopedagógico con el enfoque de la acción posibilita tener en cuenta el contexto de cada estudiante y su participación en diversos espacios, para dar respuestas a distintas situaciones. Al aplicar el método de la acción, cada estudiante es el actor social fundamental de su propio proceso de aprendizaje. Se convierte en soporte para los demás usuarios, en el marco de un ambiente adecuado y confortable que permita el trabajo de construcción, colectiva e individual, de conocimiento, tal como lo expresa Claire Bourguignon [24]. Con todo, siendo el aprendizaje una función compleja multivariable, es necesario continuar observando los modelos existentes, retroalimentando los mismos y creando nuevo material didáctico para fortalecer las competencias lingüísticas en inglés.

\section{Referencias}

[1] M. Agudelo, Importancia del Diseño Instruccional en Ambientes Virtuales de Aprendizaje, Medellín, Colombia: Universidad de Antioquia, 2009.

[2] J. P. Cerro Martínez, Tendencias y Aplicaciones de las Tic dentro del ámbito educativo, Barcelona: Universidad Oberta de Catalunya - Elearn Center, 2015.

[3] [3] B. Tomlinson, Materials development in language teaching, Segunda Edición ed., Cambridge: Cambridge University Press, 2011, p. 451

[4] J. Stephenson, Modelos pedagógicos y de e-learning, Barcelona: Universidad Oberta de Catalunya, 2015.

[5] Convenio de asociación E-Learning 2.0 Colombia, "Propuesta de Metodología para transformar programas presenciales a virtuales o E-Learning”, Bucaramanga: Ministerio de Educación Nacional, 2007.

[6] Ministerio de Educación Nacional de Colombia, MEN, 2006

[7] K. Schwienhorst, «Why Virtual, Why Environments? Implementing Virtual Reality Concepts in ComputerAssisted Language Learning,» Simulation \& Gaming, vol. 33, nº 2, pp. 196-209, 2002.

[8] M. Slater, A. Sadagic, M. Usoh y R. Schroeder, «Small-Group Behavior in a Virtual and Real Environment: A Comparative Study,» Presence: Teleoperators and Virtual Environments, vol. 9, n 1, pp. 37-51, 2000.

[9] J. Visser, «Changing learning environments: The real and not so real of reality and virtuality,» 1999. [En línea]. Available: http://www.unesco.org/education/educprog/lwf/dl/virtual.pdf. 
[10] M. Driessen, J. van Emmerik, K. Fuhri, L. Nygren-Junkin y M. Spotti, «Use in L2 Education for Adult Migrants: A qualitative study in the Netherlands and Sweden,» The Institute for Prospective Technological Studies., 2011.

[11] L. Miranda, C. Morais y P. Dias, «Pedagogical Approaches for Online Environments,» de Computers and Education: Towards Educational Change and Innovation, A. J. Mendes, I. Pereira y R. Costa, Edits., 2008, pp. 91-102.

[12] A. Fernández-Pampillón Cesteros, «Las plataformas E-learning para la enseñanza y el aprendizaje universitario en Internet,» de Las plataformas de aprendizaje. Del mito a la realidad., Madrid, Universidad Complutense de Madrid, 2009, pp. 45-73.

[13] M. Tomé, «La enseñanza y aprendizaje del francés lengua extranjera en Internet,» 1998.

[14] L. V. Marulanda y M. I. Berdugo Torres, «Un estado del arte del desarrollo de ambientes web instruccionales para lenguas y otras áreas,» Revista Lenguaje, vol. 33, pp. 129-158, 2005.

[15] D. C. Arguelles Pabón y N. Nagles García, «Estrategias para promover procesos de aprendizaje autónomo,» 19 09 2012. [En línea]. Available: http://bloge-portafoliojorge.blogspot.com.co/search?q=se+puede+decir+como. [Último acceso: 2002 2017].

[16] UNESCO, «Conferencia Mundial sobre la Educación Superior. La educación superior en el siglo XXI. Visión y acción,» UNESCO, Paris, 1998.

[17] UNESCO, «Informe mundial sobre el aprendizaje y la educación de adultos,» Instituto de la UNESCO para la Educación a lo Largo de Toda la Vida., Alemania, 2010.

[18] F. Y. Mesa Jiménez y Á. M. Soler Fonseca, «Políticas públicas en tecnologías de la información y la comunicación y su incidencia en la universidad colombiana (2000-2008),» de Memorias del Congreso de Investigación y Pedagogía, II Nacional, I Internacional, Tunja, Colombia, 2011.

[19] Departamento Nacional de Planeación, «Conpes 3072. Agenda de Conectividad,» Bogotá, 2000.

[20] G. Tibaná Herrera, «Gestión de contenidos educativos en educación superior, en Objetos de Aprendizaje: prácticas y perspectivas educativas,» Pontificia Universidad Javeriana, Bogotá, 2009.

[21] G. Davies, «CALL (computer assisted language learning),» 2016. [En línea]. Available:

https://www.llas.ac.uk/resources/gpg/61. [Último acceso: 2002 2017].

[22] Wikipedia, «Enseñanza de Lenguas Asistida por Ordenador. Levy (1997),» 2015. [En línea].

[23] Colombia aprende, «Colombia Aprende,» Ministerio de Educación Nacional, 1611 2016. [En línea]. Available: http://aprende.colombiaaprende.edu.co/ckfinder/userfiles/files/Libro\%20Innovacion\%20MEN\%20-\%20V2.pdf. [Último acceso: 2002 2017].

[24] C. Bourguignon, «De l'approche communicative à l'approche communic'actionnelle : une rupture épistémologique en didactique des langues-cultures,» Synergie Europe, vol. 1I, 2006.

\section{Sobre los autores}

\section{Olga Andrea Pedraza Rojas}

Licenciada en Lenguas Modernas de la Pontificia Universidad Javeriana, Bogotá, Colombia. Estudiante de la Maestría en E-Learning de la Universidad Autónoma de Bucaramanga en convenio con la Universidad Oberta de Catalunya. Cúcuta, Colombia.

\section{Miguel Francisco Crespo Alvarado}

Candidatura Doctoral en Ciencias Aplicadas, Mención Sistemología Interpretativa, Universidad de los Andes, Venezuela. Maestría en Sistemología Interpretativa, Universidad de los Andes, Venezuela, 2005. Maestría en Educación, Universidad Autónoma de la Laguna, México, 2001. Maestría en Administración, Universidad Autónoma de la Laguna, 1994. Licenciatura en Administración de Recursos Humanos, Universidad Autónoma de la Laguna. 1991. Ciudad de México, México D.F. 